\title{
CHEMICAL IMMOBILIZATION AND ANESTHESIA OF FREE-LIVING AARDVARKS (ORYCTEROPUS AFER) WITH KETAMINE-MEDETOMIDINE-MIDAZOLAM AND ISOFLURANE
}

\author{
Benjamin Rey, ${ }^{1,4,5}$ Mary-Ann Costello, ${ }^{2}$ Andrea Fuller, ${ }^{1}$ Anna Haw, ${ }^{1}$ Robyn S. Hetem, ${ }^{1}$ \\ Duncan Mitchell, ${ }^{1}$ and Leith C. R. Meyer ${ }^{1,3}$ \\ ${ }^{1}$ Brain Function Research Group, School of Physiology, Faculty of Health Sciences, University of the Witwatersrand, \\ 7 York Road, Parktown, Johannesburg 2193, South Africa \\ ${ }^{2}$ Central Animal Service, University of the Witwatersrand, 7 York Road, Parktown, Johannesburg 2193, South Africa \\ ${ }^{3}$ Department of Paraclinical Science, Faculty of Veterinary Science, University of Pretoria, Onderstepoort 0110, South Africa \\ ${ }^{4}$ Current address: Laboratoire de Biométrie et Biologie Evolutive, CNRS-UMR5558 Université Lyon1, 43 bvd 11 \\ novembre 1918, F-69622 Villeurbanne cedex, France \\ ${ }^{5}$ Corresponding author (email: benjamin.rey@univ-lyon1.fr)
}

ABSTRACT: We evaluated the effectiveness of a ketamine-medetomidine-midazolam drug combination administered intramuscularly by remote injection followed by isoflurane anesthesia in freeliving aardvarks (Orycteropus afer). Seven aardvarks weighing 33-45 kg were immobilized to perform surgical implantation of temperature loggers using $3.8 \mathrm{mg} / \mathrm{kg}$ ketamine, $0.1 \mathrm{mg} / \mathrm{kg}$ medetomidine, and $0.25 \mathrm{mg} / \mathrm{kg}$ midazolam. Immobilized aardvarks were transported to a surgical theater and received $0.5-1 \%$ isoflurane in oxygen after tracheal intubation. After surgery, medetomidine was antagonized with $0.5 \mathrm{mg} / \mathrm{kg}$ atipamezole, and aardvarks were released at the site of capture. We recorded induction and recovery times, clinical and physiologic parameters, and conducted blood gas analyses before and during isoflurane administration. Aardvarks showed initial effects within 3 min and reached lateral recumbency within 7 min after drug administration. Heart rate (50-67 beats/min), respiratory rate (10-15 breaths/min), oxygen hemoglobin saturation $\left(\mathrm{SaO}_{2} ; 90-97 \%\right)$, and rectal temperature (34.0-37.5 C) were within acceptable physiologic ranges. Mean arterial blood pressure was initially high $(146 \pm 12 \mathrm{mmHg})$, but the hypertension resolved over time. Rectal temperature dropped significantly during anesthesia. Four animals had to be treated to relieve apnea. Blood gas analyses revealed mild to moderate hypercapnia but no hypoxaemia. The ketamine-medetomidine-midazolam combination provided effective immobilization. Combined with a low concentration of isoflurane, it can be used for prolonged surgical procedures in wild aardvarks. However, caution is needed, and monitoring of clinical parameters is required.

Key words: Aardvark anesthesia, remote injection, chemical immobilization, cardiovascular, respiratory and body temperature monitoring.

\section{INTRODUCTION}

Biologic investigations of free-living aardvarks (Orycteropus afer) are hampered by the animals' nocturnal and elusive lifestyle. Improving methods of capture and longduration anesthesia is necessary to implement management actions and investigate the behavior, ecology, and physiology of aardvarks in their natural habitat (Van Aarde et al. 1992; Taylor and Skinner 2004). This work is part of a study requiring the capture of aardvarks and implantation of data loggers into the abdominal cavity.

Aardvark capture techniques include hand capture, setting funnel traps at burrow entrances, and netting, followed by intramuscular (IM) injection of immobilizing agents (van Aarde et al. 1992; Nel et al. 2000). It is possible that these methods of capture elicit a severe stress response as mortalities have been reported (Taylor and Skinner 2003). Projectile darts provide a means of remotely injecting immobilizing drugs, thus reducing the stress response to capture (Boesch et al. 2011). However, remote injection techniques have not been used in wild aardvarks, despite their effective use for animals in captivity (Langan 2008).

The drug cocktails for immobilization of captive aardvarks generally include ketamine combined with medetomidine, detomidine, diazepam, xylazine, or 
droperidol and fentanyl (Stetter 2003; Langan 2008). For trapped and restrained wild aardvarks, ketamine has also been used with medetomidine, midazolam, or xylazine with various levels of success (van Aarde et al. 1992; Nel et al. 2000), with the combination of ketamine and medetomidine being the most effective.

If long-term surgical procedures are planned, anesthesia may be prolonged with volatile anesthetic agents (Stetter 2003; Langan 2008). Simple clinical parameters, such as heart rate and respiratory rate, have been reported; however, as far as we know, no other clinical parameters have been described for immobilized aardvarks. We tested the effectiveness of projectile darts for immobilizing free-ranging aardvarks using a combination of ketamine, medetomidine, and midazolam and recorded clinical parameters following capture and during prolonged isoflurane anesthesia.

\section{MATERIALS AND METHODS}

The study took place in July 2012 at Tswalu Kalahari Game Reserve $\left(27^{\circ} 14^{\prime} 57^{\prime \prime} \mathrm{S}\right.$, $22^{\circ} 22^{\prime} 52^{\prime \prime} \mathrm{E}, 1,150-\mathrm{m}$ elevation). All procedures received approval from the Department of Environment and Nature Conservation (Northern Cape, South Africa) and the Animal Ethics Screening Committee of the University of the Witwatersrand (certificate 4 October 2011).

\section{Chemical immobilization}

Seven aardvarks were spotted from a vehicle and approached on foot from downwind (darting time was between 3:30 PM and 6:30 PM). We used 2-mL darts (Type P, 32-mm needle, Pneu-dart, Williamsport, Pennsylvania, USA) propelled with a $\mathrm{CO}_{2}$-pressurized dart gun (Dan-Inject, Børkop, Denmark). Darts contained a 2-mm barb, except for the first aardvark, which was immobilized with a barbless dart. Darting distance was 10-25 m, and the $\mathrm{CO}_{2}$ pressure was 4.5-10 bar. Drug doses were calculated for an anticipated 50-kg aardvark. Darts were filled with $150 \mathrm{mg}$ ketamine, $4 \mathrm{mg}$ medetomidine, and $10 \mathrm{mg}$ midazolam using concentrated drugs (ketamine: $200 \mathrm{mg} / \mathrm{mL}$; medetomidine: $50 \mathrm{mg} / \mathrm{mL}$; and midazolam: $50 \mathrm{mg} / \mathrm{mL}$; Kyron Laboratories, Johannesburg, South Africa), and we added sterile water to reach a final volume of $2 \mathrm{~mL}$. We recorded the times after darting to initial effects and to lateral recumbency. Once immobilized, we placed a blindfold over the aardvark's eyes and cotton plugs in the external auditory canals. We inserted catheters (20 gauge, Introcam, Melsungen, Germany) into the marginal ear vein and auricular artery. Aardvarks were loaded onto a vehicle, placed sternal on a mattress, and transported to a surgical theater.

\section{Isoflurane anesthesia and surgical procedure}

Isoflurane (0.5-3\%, Isofor, Safe Line, South Africa) was administered in $100 \%$ oxygen via a face mask once we arrived at the surgical theater. Aardvarks were kept sternal throughout the proceedings, except during surgery when the aardvarks were positioned in lateral recumbency. Before intubation, we sprayed a topical anesthetic (xylocaine, AstraZeneca, Johannesburg, South Africa) onto the larynx. The upper jaw was held open using a piece of crepe bandage, which was placed under the hard palate. Intubation with a 6.5 to $7 \mathrm{~mm}$ endotracheal tube (Kyron Laboratories) was performed with the aid of a laryngoscope (310 mm, Millers, Surgical System, Cape Town, South Africa) and a stylet. The cuff was inflated with $5 \mathrm{~mL}$ of air.

Anesthesia was maintained with isoflurane $(0.5-1 \%)$ administered in oxygen using a circle rebreathing system. Aardvarks were placed on a heating pad (Deluxe, DisChem Pharmacies, Midrand, South Africa) and partially covered with shock blankets. We administered lactated Ringer's solution intravenously (IV; Adcock Ingram Critical Care, Johannesburg, South Africa) as maintenance fluids.

For study purposes, we inserted data loggers into the abdomen, following published procedures (Hetem et al. 2010). Briefly, the abdominal cavity was entered via a $50-\mathrm{mm}$ incision through the skin, muscle layers, and parietal peritoneum in the left paralumbar region. After placing the loggers, the muscles and skin were sutured closed. Before the start of surgery, we administered a long-acting antibiotic (6,000 IU/kg IM procaine benzylpenicillin, Duplocillin, Intervet, South Africa), a nonsteroidal anti-inflammatory drug $(0.5 \mathrm{mg} / \mathrm{kg}$ of subcutaneous meloxicam, Metacam, Boehringer Ingelheim, Johannesburg, South Africa) and a local anesthetic agent $(5 \mathrm{~mL}$ lignocaine, Bayer, South Africa) along the incision site. At the end of the procedures, we administered an opioid analgesic $(0.01 \mathrm{mg} / \mathrm{kg}$ IM buprenorphine, Temgesic, MSD, Johannesburg, South Africa). If prolonged (>1 min) apnea occurred, we administrated doxapram (40 mg IV Dopram, Bodene, Port Elizabeth, South Africa) if the aardvark was 
not intubated, or we provided positive pressure ventilation in an intubated aardvark by gentle compression of the anesthetic reservoir bag, while monitoring thoracic excursions.

Aardvarks were supplemented with $100 \%$ oxygen during transportation back to the capture site. Surrounding areas were checked for potential predators before the animals were released. For reversal, we injected atipamezole slowly IV $(0.5 \mathrm{mg} / \mathrm{kg}$; Antisedan, Pfizer Laboratories, Johannesburg, South Africa). Catheters were removed, and hemostasis was achieved by direct pressure. The endotracheal tube was removed when the aardvark showed signs of arousal. We recorded the time to first movement and the time to standing after atipamezole administration.

\section{Clinical patient monitoring and data recording}

Throughout the anesthetic procedure, we regularly assessed depth of anesthesia by monitoring muscle tone, palpebral reflex, pedal withdrawal reflex, and changes in respiratory rate and blood pressure. Clinical data were recorded every $5 \mathrm{~min}$ until the end of the procedure. Respiratory rate was measured by observing thoracic excursions, arterial oxygen hemoglobin saturation $\left(\mathrm{SpO}_{2}\right)$, and pulse rate (assumed to be similar to heart rate), with a pulse oximeter (Nonin Medical $9847 \mathrm{~V}$, Plymouth, Minnesota, USA) using a lingual clip, and rectal temperature with a thermocouple probe inserted $100 \mathrm{~mm}$ into the rectum. A sidestream capnograph (LS1-10R, Medair, Fibervägen, Sweden) was connected to the endotracheal tube and recorded endtidal $\mathrm{CO}_{2}\left(\mathrm{EtCO}_{2}\right)$. A pressure transducer (DPT-200, Utah Medical Products, Midvale, Utah, USA) was connected to the arterial catheter and served to record systolic, diastolic, and mean arterial blood pressures.

Arterial blood was collected anaerobically into $1-\mathrm{mL}$ heparinized syringes and analyzed using a blood gas analyzer (Roche OPTI CCA, Kat Medical, Johannesburg, South Africa) to determine $\mathrm{pH}$, partial pressure of carbon dioxide $\left(\mathrm{PaCO}_{2}\right)$, partial pressure of oxygen $\left(\mathrm{PaO}_{2}\right)$, bicarbonate concentration, and $\mathrm{SaO}_{2}$. The first blood sample was collected before oxygen and isoflurane administration $\left(T_{1}\right.$, 30 min after darting). The next three blood samples $\left(\mathrm{T}_{2}\right.$ to $\left.\mathrm{T}_{4}\right)$ were collected $30 \mathrm{~min}, 1 \mathrm{hr}$, and $2 \mathrm{hr}$ after oxygen and isoflurane administration commenced. Blood gases were measured and reported at a temperature of $37 \mathrm{C}$.

\section{Statistical analysis}

Blood gas values measured at times $T_{2}$ to $T_{4}$ were compared with those measured at time $\mathrm{T}_{1}$ by paired $t$-tests with Bonferroni correction. We investigated the changes of each clinical parameter over time by fitting generalized additive mixed models to account for the possible nonlinear effect of the variable "time." These models allow for dealing with high interindividual heterogeneity in the duration of anesthesia (the duration of anesthesia was not unnecessarily prolonged to allow extra measurements to be taken). We entered a categorical variable "individuals" as a random factor to account for repeated measures. Tested clinical parameters were: heart rate, $\mathrm{SpO}_{2}$, intra-arterial blood pressure, respiratory rate, rectal temperature, and $\mathrm{EtCO}_{2}$. Models are presented with their 95\% confidence interval for each of the parameters that showed a significant trend over time. Statistical analyses were performed with $\mathrm{R}$ software (R Development Core Team 2010). $P<0.05$ was considered a significant difference. Results are presented as mean $\pm \mathrm{SD}$.

\section{RESULTS}

\section{Chemical immobilization}

We successfully darted seven aardvarks weighing $39.6 \pm 3.9 \mathrm{~kg}$ (range, 33-45 kg). The mean drug dose administered (excluding the aardvark darted twice) was $3.8 \mathrm{mg} / \mathrm{kg}$ ketamine (range $=3.3-4.6), 0.10 \mathrm{mg} / \mathrm{kg}$ medetomidine (range $=0.09-0.12$ ) and 0.25 $\mathrm{mg} / \mathrm{kg}$ of midazolam (range $=0.22-0.30$ ). The darts discharged in the gluteal muscles of all aardvarks, except for one dart that penetrated into the panniculus muscle in the caudal area of the left thorax. The aardvarks showed a minor reaction to the penetration of the darts; typically, they ran 10-30 m before resuming foraging behavior. The chronology of events related to immobilization and anesthesia, including induction and recovery times, is presented in Table 1 . The first aardvark, weighing $39 \mathrm{~kg}$, was darted with a barbless needle. Although the dart hit the gluteal region, it immediately bounced out. Immobilization was not deep enough to ensure safe handling and transport; therefore, we administered a second dart containing $2.25 \mathrm{mg} / \mathrm{kg}$ of ketamine, $0.06 \mathrm{mg} / \mathrm{kg}$ of medetomidine, and $0.15 \mathrm{mg} / \mathrm{kg}$ of midazolam (data for induction times were excluded for this aardvark). Of the seven aardvarks, only one went into a burrow. This 
TABLE 1. Chronology of induction, anesthesia, and recovery of aardvarks (Orycteropus afer) immobilized with ketamine, medetomidine, and midazolam, and maintained under surgical anesthesia with isoflurane. At release, the effects of medetomidine were reversed with atipamezole. Values are expressed in minutes for six (induction time) or seven (recovery times) independent observations.

\begin{tabular}{lcc}
\hline & Mean \pm SD & Range \\
\hline Time to initial signs (minutes after darting) & $3.1 \pm 1.1$ & $2-5$ \\
Time to lateral recumbency (minutes after darting) & $3.4 \pm 2.5$ & $19-35$ \\
Duration of transport (minutes) & $25 \pm 5$ & $35-60$ \\
Time of isoflurane administration (minutes after darting) & $53 \pm 9$ & $45-82$ \\
Time of intubation (minutes after darting) & $63 \pm 12$ & $73-166$ \\
Duration of isoflurane administration (minutes) & $129 \pm 34$ & $79-144$ \\
Time to start of surgery (minutes after darting) & $96 \pm 21$ & $36-72$ \\
Duration of surgery (minutes) & $56 \pm 13$ & $13-34$ \\
Return transport time (minutes) & $21 \pm 8$ & $172-258$ \\
Time of atipamezole administration (minutes after darting) & $20 \pm 34$ & $3-6$ \\
Time to first voluntary movement (minutes after reversal) & $2.1 \pm 1.9$ & $3.1 \pm 1.9$ \\
Time to standing (minutes after reversal) & $206 \pm 34$ & $177-262$ \\
Total immobilization time (minutes) & & \\
\hline
\end{tabular}

animal was left undisturbed for $6 \mathrm{~min}$ to allow the drugs to take effect, then rapidly retrieved by digging into the burrow.

\section{Clinical data}

Clinical parameters measured $30 \mathrm{~min}$ after darting $\left(\mathrm{T}_{1}\right)$ are presented in Table 2. At $\mathrm{T}_{1}$, aardvarks were bradycardic, hypertensive, and had low rectal temperatures. Figure 1 shows significant changes over time in heart rate $(P=0.003), \mathrm{SpO}_{2}(P<0.001)$, systolic, mean, and diastolic arterial blood pressure $(P<0.001$ for each pressure), and rectal temperature $(P<0.001)$. Heart rate decreased for the first $2 \mathrm{hr}$ after darting but tended to increase at the end of surgery (Fig. 1A). $\mathrm{SpO}_{2}$ increased over time and remained above 95\% throughout surgery (Fig. 1B). Blood pressure and rectal temperature decreased over time (Fig. 1C, 1D).
Changes in respiratory rate $(P=0.94)$ and $\mathrm{EtCO}_{2}(P=0.65)$ were not statistically significant. However, two aardvarks became apneic 6 to $10 \mathrm{~min}$ after receiving concentrations of $2-3 \%$ isoflurane via a mask. Following intubation, one aardvark stopped breathing after receiving isoflurane for $18 \mathrm{~min}$. Another aardvark stopped breathing during transport back to the field. Concentrations higher than $1 \%$, isoflurane caused a substantial drop in blood pressure, so surgical anesthesia was maintained with low isoflurane concentration. Three of the aardvarks reacted during surgery; in these aardvarks, the isoflurane was increased temporarily for a few minutes.

\section{Blood gas analyses}

Blood gas analyses values are given in Table 3. At $\mathrm{T}_{1}, \mathrm{PaO}_{2}$ and $\mathrm{SaO}_{2}$ indicated

TABLE 2. Clinical parameters measured from seven aardvarks (Orycteropus afer) immobilized with ketamine, medetomidine, and midazolam at time $\mathrm{T}_{1}$ (30 minutes after darting), before oxygen and isoflurane administration.

\begin{tabular}{lcc}
\hline \multicolumn{1}{c}{ Clinical parameters at time $\mathrm{T}_{1}$} & Mean $\pm \mathrm{SD}$ & Range \\
\hline Heart rate (pulse per minute) & $60 \pm 7$ & $50-67$ \\
Respiratory rate (breaths per minute) & $12 \pm 2$ & $10-15$ \\
Arterial oxygen hemoglobin saturation $(\%)$ & $93 \pm 2$ & $90-97$ \\
Systolic blood pressure (mmHg) & $162 \pm 10$ & $154-174$ \\
Mean blood pressure (mmHg) & $146 \pm 12$ & $134-163$ \\
Diastolic blood pressure (mmHg) & $138 \pm 13$ & $125-158$ \\
Rectal temperature $(\mathrm{C})$ & $35.5 \pm 1.5$ & $34.0-37.5$ \\
\hline
\end{tabular}




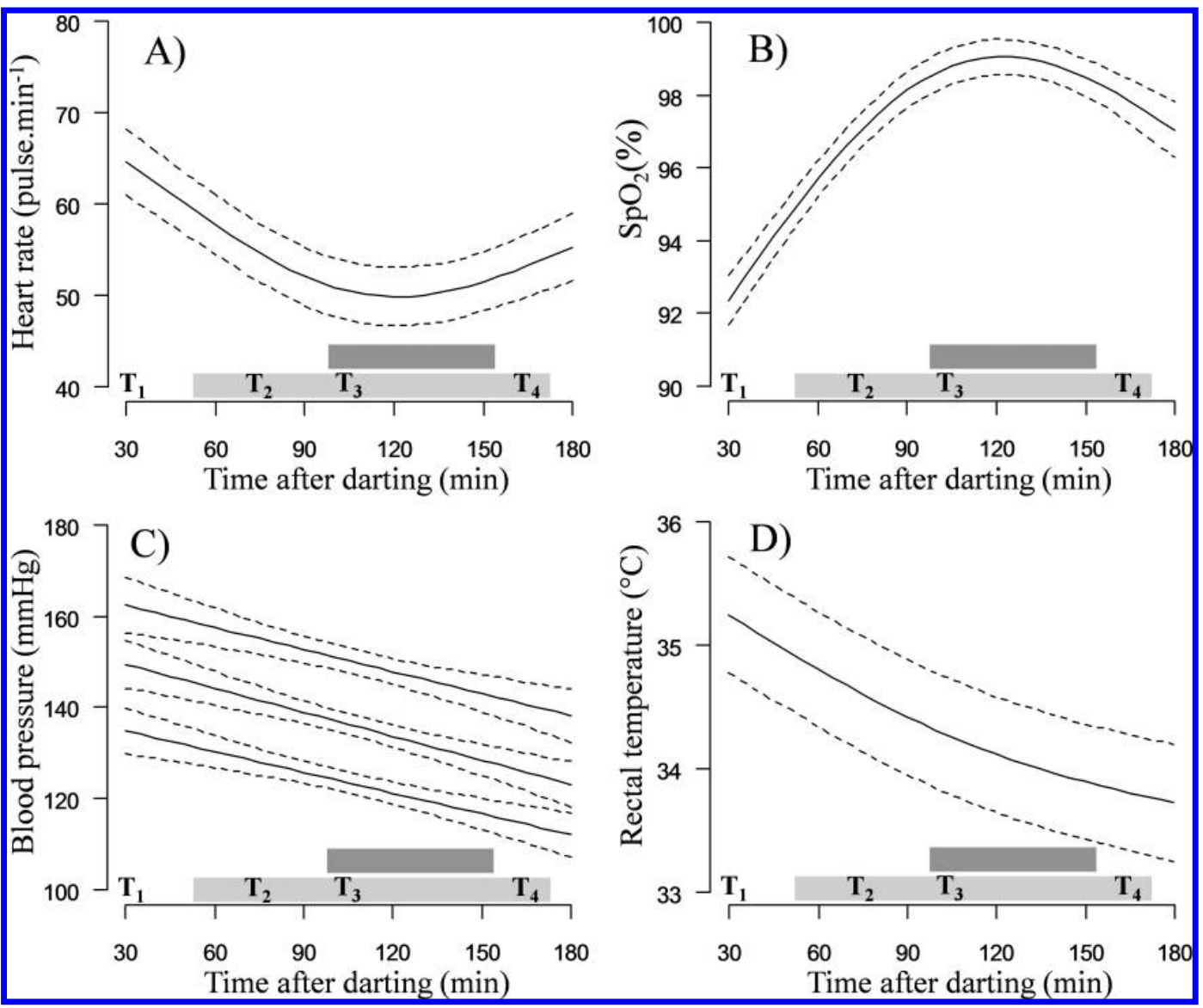

Figure 1. Changes over time in (A) heart rate; (B) peripheral oxygen hemoglobin saturation; (C) systolic, mean, and diastolic arterial blood pressure; and (D) rectal temperature in seven aardvarks (Orycteropus afer) immobilized with ketamine, medetomidine, and midazolam and maintained under isoflurane anesthesia. Solid lines represent the prediction of general additive mixed-effects models fitted on clinical data recorded every 5 min; dashed lines represent the $95 \%$ confidence intervals. Light grey bars represent time of isoflurane inhalation; dark grey bars indicate surgery time. $\mathrm{T}_{1}$ to $\mathrm{T}_{4}$ represent the times at which blood gas analyses were performed.

satisfactory, but not complete blood oxygenation. Both $\mathrm{PaO}_{2}$ and $\mathrm{SaO}_{2}$ increased significantly with oxygen and isoflurane administration. Aardvarks showed mild to moderate hypercapnia. Hypercapnia together with relatively low $\mathrm{PaO}_{2}$ at time $\mathrm{T}_{4}$ were mainly explained by one aardvark exhibiting respiratory depression at the time of blood sampling. Bicarbonate increased significantly at time $\mathrm{T}_{4} ; \mathrm{pH}$ values did not indicate acidemia.

\section{Recovery}

Mean recovery times after atipamezole administration are shown in Table 1. After reversal, first movements were recorded within $2 \mathrm{~min}$; the aardvarks were standing within 5 min and rapidly walked away, with an ataxic gait. Because the aardvarks were released at night, the time at which they were no longer ataxic was not recorded. Aardvarks were tracked and observed periodically for 6 mo after surgery; they displayed normal behavior.

\section{DISCUSSION}

The ketamine-medetomidine-midazolam combination administered intramuscularly with $\mathrm{CO}_{2}$-propelled darts had a 
TABLE 3. Acid-base and respiratory characteristics of arterial blood gas from seven aardvarks (Orycteropus afer) immobilized with ketamine, medetomidine, and midazolam before (sample $\mathrm{T}_{1}$ ) and after receiving oxygen and isoflurane (sample $\mathrm{T}_{2}$ to $\mathrm{T}_{4}$ ). Values are expressed as mean $\pm \mathrm{SD}$. We were unable to perform blood gas analysis for two aardvarks at $\mathrm{T}_{4}$, so data are presented for five aardvarks. ${ }^{a}$

\begin{tabular}{lcccc}
\hline & Sample $\mathrm{T}_{1}$ & Sample $\mathrm{T}_{2}$ & Sample $_{3}$ & Sample $_{4}$ \\
\hline Time after darting (minutes) & $(29 \pm 11)$ & $(62 \pm 12)$ & $(98 \pm 10)$ & $(173 \pm 11)$ \\
$\mathrm{pH}$ & $7.35 \pm 0.05$ & $7.35 \pm 0.07$ & $7.35 \pm 0.04$ & $7.33 \pm 0.03$ \\
$\mathrm{PaCO}_{2}(\mathrm{mmHg})$ & $47 \pm 5$ & $53 \pm 6$ & $53 \pm 4$ & $57 \pm 4^{\mathrm{b}}$ \\
$\mathrm{PaO}_{2}(\mathrm{mmHg})$ & $84 \pm 13$ & $147 \pm 58^{\mathrm{b}}$ & $457 \pm 54^{\mathrm{b}}$ & $340 \pm 176$ \\
$\mathrm{HCO}_{3-}(\mathrm{mmol} / \mathrm{L})$ & $25.4 \pm 3.4$ & $28.5 \pm 3.0$ & $28.9 \pm 1.7$ & $29.4 \pm 1.3^{\mathrm{b}}$ \\
$\mathrm{SaO}_{2}(\%)$ & $95 \pm 3$ & $99 \pm 1^{\mathrm{b}}$ & $100 \pm 0^{\mathrm{b}}$ & $99 \pm 1^{\mathrm{b}}$ \\
\hline
\end{tabular}

${ }^{\text {a }} \mathrm{PaCO}_{2}=$ partial pressure of carbon dioxide; $\mathrm{PaO}_{2}=$ partial pressure of oxygen; $\mathrm{HCO}_{3-}=$ bicarbonate concentration; $\mathrm{SaO}_{2}=$ oxygen hemoglobin saturation.

${ }^{\mathrm{b}}$ Values statistically different compared with $\mathrm{T}_{1}$.

rapid onset of action and was effective for immobilization of free-living aardvarks. Prolongation of anesthesia was easily provided by the administration of a low concentration of isoflurane in oxygen after endotracheal intubation. Administration of atipamezole led to a quick recovery, and aardvarks rapidly walked away.

The major risk of remote injection is losing contact with a darted aardvark, which may have dire consequences if an aardvark is exposed to predators or if it lies in an awkward position, particularly in a burrow, which may result in respiratory compromise (Nel et al. 2000). By immobilizing aardvarks when they were actively foraging, most became recumbent on the ground surface. One aardvark entered a burrow after being darted but was excavated without mishap. To reduce the risk of losing a darted aardvark, the use of tracking-transmitter darts could be considered. Remote injection may also cause injuries if the location of the dart is inappropriate. In our study, one dart hit the thoracic region but fortunately caused no damage. Another inherent risk of remote injection is overdosing an animal because of incorrect estimation of body mass. The aardvarks we immobilized were on average $20 \%$ lighter than expected.

The combination of ketamine, medetomidine, and midazolam, at an overestimated dose, induced quick immobilization. The induction time was similar to that observed in captive aardvarks given ketamine in combination with detomidine (Vodicka 2004) and in wild but restrained aardvarks given ketamine in combination with medetomidine or xylazine ( $\mathrm{Nel}$ et al. 2000). The addition of midazolam did not appear to shorten the time to recumbency. However, because the pharmacokinetic and the pharmacodynamic of the immobilizing drugs are likely to be affected by animal stress levels (Antonia et al. 2012), a direct comparison between induction times in these different studies is likely invalid.

We believe that midazolam increased muscle relaxation and improved sedation, thereby improving immobilization. Combined with the potent minimum alveolar concentration (MAC) sparing effects of medetomidine and hypothermia (Satas et al. 1996), the addition of midazolam may have also contributed to the reduction in the MAC of isoflurane. On the whole, we found that a low isoflurane concentration $(0.5-1 \%)$ was adequate for surgical anesthesia in the aardvark.

The effect of the immobilizing drugs was also assessed by monitoring physiologic parameters. As far as we are aware, we are the first to report arterial blood pressures in aardvarks, so no comparison could be made with other studies. Allometric equations for cardiovascular parameters predict that a $33-$ to $45-\mathrm{kg}$ mammal should have a systolic and 
diastolic blood pressure of approximately 140 and $95 \mathrm{mmHg}$, respectively, and a heart rate of 95-100 beats/min (Seymour and Blaylock 2000). Aardvarks were, thus, hypertensive and bradycardic in our study (Table 2). Hypertension has been observed in other species given the same combination of drugs (Curro et al. 2004; Ebner et al. 2007; Schernthaner et al. 2011) and is attributed mainly to the effects of medetomidine on the peripheral vasculature. Bradycardia, which has been observed in aardvarks (Nel et al. 2000; Langan 2008), could also be attributed to the alpha-2 agonists cardiovascular effects, primarily as a secondary effect of druginduced vasoconstriction, but it may also reflect the animal's naturally low metabolic rate (McNab 1979).

Supplementing the immobilizing agents with a low isoflurane concentration provided adequate surgical-level anesthesia. However, apneas occurred in four aardvarks, mainly after isoflurane concentration was temporarily increased. The loss of voluntary respiration in these aardvarks could possibly be attributed to sensitivity to isoflurane. Alternatively, midazolam and medetomidine could have exacerbated isoflurane-induced respiratory depression and possibly decreased ventilatory response to $\mathrm{CO}_{2}$ (Tranquilli et al. 2007).

During anesthesia, blood pressure fell linearly, with systolic pressure approaching that expected of conscious mammals of similar mass. However, diastolic blood pressure remained well above its allometric target, suggesting that the peripheral vasoconstriction was present several hours after darting. Heart rates fell progressively during anesthesia, with some rebound during surgery possibly due to intraoperative stimulation or a decrease in blood pressure, leading to a normalization of heart rate.

By using the alveolar gas equation (Martin 1999), we calculated that the $\mathrm{PaO}_{2}$ of aardvarks breathing ambient air at an altitude of $1,150 \mathrm{~m}$ would be about $80 \mathrm{mmHg}$, which is close to the actual $\mathrm{PaO}_{2}$ we measured after immobilization
(Table 3). When the aardvarks were breathing isoflurane in $100 \%$ oxygen, calculated $\mathrm{PaO}_{2}$ was $570 \mathrm{mmHg}$, but the measured $\mathrm{PaO}_{2}$ was highly variable, and did not approach this calculated value (Table 3 ). This finding indicates incomplete equilibration of arterial gas with alveolar gas possibly due to hypoventilation, ventilation-perfusion mismatching, shunting, or other pathophysiologic cardiorespiratory changes (Martin 1999). Nevertheless, the increase in inspired oxygen did improve blood oxygenation and increased the $\mathrm{SpO}_{2}$ to 99$100 \%$ at times $\mathrm{T}_{2}$ to $\mathrm{T}_{4}$ (Table 3 ). The $\mathrm{PaCO}_{2}$ values indicate mild to moderate hypercapnia, and this may be related to anesthetic-induced respiratory depression. The concentration of bicarbonate increased sufficiently to prevent respiratory acidosis; blood $\mathrm{pH}$ remained close to normal.

Despite the aardvarks being placed on heating blankets, their body temperatures decreased during prolonged anesthesia, confirming previous observations ( Nel et al. 2000). The aardvarks were released with a temperature about $1.5 \mathrm{C}$ lower than that at capture, an undesirable outcome, even though their temperature remained within the normal range for conscious aardvarks (Taylor and Skinner 2004). Reducing heat loss with blankets and insulated mattresses during transport and surgery, maintaining warm ambient temperatures in the theater, and infusing prewarmed fluids should be instituted.

At the end of surgery, after isoflurane administration was stopped, residual immobilization was adequate to allow for transport of the aardvarks back to the field with little spontaneous movement or arousal. Such residual immobilization probably resulted from a slow recovery from prolonged isoflurane anesthesia, which might have been exacerbated by hypothermia. The residual immobilization was quickly reversed by atipamezole, which could mean that either medetomidine was still effective at the time of reversal or that arousal occurred as a result of an increased noradrenergic effect from alpha-2 adrenergic receptor 
antagonism (Pertovaara et al. 2005). Importantly, the recovery was smooth, and excitement did not occur.

In summary, we have established that darting is effective for chemical immobilization of free-living aardvarks, allowing for new management and research opportunities in this relatively understudied animal. The ketamine-medetomidine-midazolam combination induced a short induction time, good immobilization, and little adverse effects on the cardiorespiratory systems. The addition of low concentrations of isoflurane was sufficient to maintain prolonged surgical anesthesia. However, depth of anesthesia, body temperature, and respiration need to be closely monitored, particularly as animals easily become hypothermic, and there may be sporadic incidences of arousal and apnea.

\section{ACKNOWLEDGMENTS}

We thank the Claude Leon Foundation, the University of the Witwatersrand, and the South African National Research Foundation for funding. We are grateful to the Tswalu Foundation for support, to E. Oppenheimer \& Son for permission to undertake this work at the Tswalu Kalahari Game Reserve, and to the Department of Tourism, Environment and Conservation (Northern Cape) for their authorization. We thank Duncan MacFadyen, Gus van Dyk, and Dylan Smith for their support with data collection and the two anonymous referees for their constructive comments on the manuscript.

\section{LITERATURE CITED}

Antonia K, Anastasia A, Tesseromatis C. 2012. Stress can affect drug pharmacokinetics via serum/ tissues protein binding and blood flow rate alterations. Eur J Drug Metab Pharmacokinet 37:1-7.

- Boesch JM, Boulanger JR, Curtis PD, Erb HN, Ludders JW, Kraus MS, Gleed RD. 2011. Biochemical variables in free-ranging whitetailed deer (Odocoileus virginianus) after chemical immobilization in clover traps or via grounddarting. I Zoo Wildl Med 42:18-28.

Curro TG, Okeson D, Zimmermann D, Armstrong DL, Simmons LG. 2004. Xylazine-midazolamketamine versus medetomidine-midazolamketamine anesthesia in captive Siberian tigers (Panthera tigris altaica). J Zoo Wildl Med 35:320-327.
Ebner J, Wehr U, Busch R, Erhardt W, Henke J. 2007. A comparative clinical study of three different dosages of intramuscular midazolammedetomidine-ketamine immobilization in cats. I Vet Med A 54:418-423.

Hetem RS, Strauss WM, Fick LG, Maloney SK, Meyer LC, Shobrak M, Fuller A, Mitchell D. 2010. Variation in the daily rhythm of body temperature of free-living Arabian oryx (Oryx leucoryx): Does water limitation drive heterothermy? J Comp Physiol B 180:1111-1119.

Langan J. 2008. Tubulidentata and Pholidota. In: Zoo animal and wildlife immobilization and anesthesia, West G, Heard D, Caulkett N, editors. Blackwell Publishing, Ames, Iowa, pp. 355358.

Martin L. 1999. All you really need to know to interpret arterial blood gases. Lippincott Williams \& Wilkins, Baltimore, Maryland, 254 pp.

McNab BK. 1979. The influence of body size on the energetic and distribution of fossorial and burrowing mammals. Ecology 60:1010-1021.

Nel PJ, Taylor A, Meltzer DGA, Haupt MA. 2000. Capture and immobilization of aardvark (Orycteropus afer) using different drug combinations. J S Afr Vet Assoc 71:58-63.

Pertovaara A, Haapalinna A, Sirviö J, Virtanen R. 2005. Pharmacological properties, central nervous system effects, and potential therapeutic applications of atipamezole, a selective $\alpha_{2}$-adrenoceptor antagonist. CNS Drug Rev 11:273-288.

R Development Core Team. 2010. R: A language and environment for statistical computing. Version 2.9.0. R Foundation for Statistical Computing, Vienna, Austria. http://www.R-project.org. Accessed June 2014.

Satas S, Haaland K, Thoresen M, Steen PA. 1996. MAC for halothane and isoflurane during normothermia and hypothermia in the newborn piglet. Acta Anaesthesiol Scand 40:452-456.

-Schernthaner A, Lendl CE, Hartmann K, Pragst I, Preissel AK, Henke J. 2011. Medetomidine/ midazolam/ketamine anaesthesia in ferrets: effects on cardiorespiratory parameters and evaluation of plasma drug concentrations. Vet Anaesth Analg 38:439-450.

Seymour RS, Blaylock AJ. 2000. The principle of Laplace and scaling of ventricular wall stress and blood pressure in mammals and birds. Physiol Biochem Zool 73:389-405.

Stetter MD. 2003. Tubulidentata. In: Zoo and wild animal medicine. 5th Ed. Fowler ME, Miller ER, editors. Elsevier Health Sciences, Philadelphia, Pennsylvania, pp. 538-541.

Taylor WA, Skinner JD. 2003. Activity patterns, home ranges and burrow use of aardvark (Orycteropus afer) in the Karoo. J Zool 261:291-297.

Taylor WA, Skinner JD. 2004. Adaptations of the aardvark for survival in the Karoo: A review. Trans $R$ Soc S Afr 59:105-108. 
Tranquilli WJ, Thurmon JC, Grimm KA, Lumb WV. 2007. Lumb and Jones' veterinary anesthesia. 4th Ed. Blackwell Publisher, Ames, Iowa, 1,057 pp. Van Aarde R, Willis C, Skinner J, Haupt MA. 1992 Range utilization by the aardvark, Orycteropus afer (Pallas, 1766) in the Karoo, South-Africa. J Arid Environ 22:387-394.
Vodicka R. 2004. Chemical immobilization of captive aardvark (Orycteropus afer). J Zoo Wildl Med 35:544-545.

Submitted for publication 13 July 2013.

Accepted 3 April 2014 